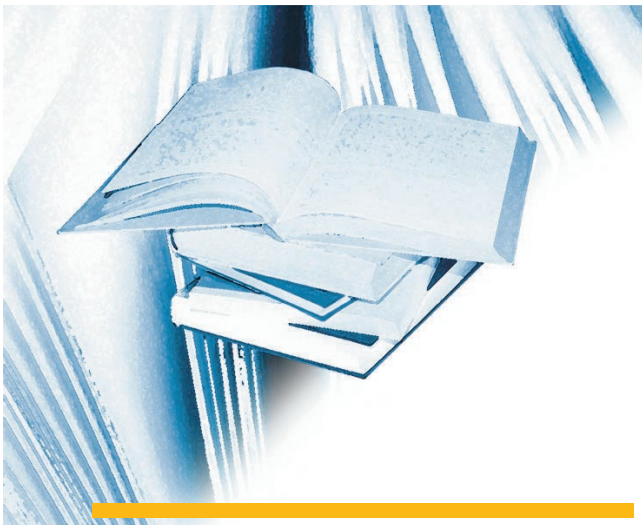

Nickel and Its Surprising Impact in Nature

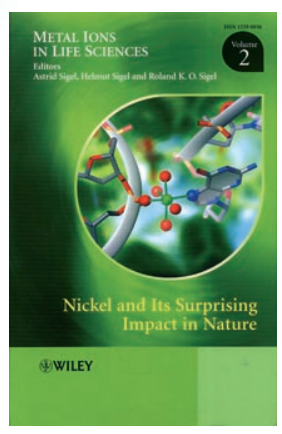

Metal lons in Life Science, Bd. 2. Herausgegeben von Astrid Sigel, Helmut Sigel und Roland K. O. Sigel. John Wiley \& Sons, Hoboken 2007. 728 S., geb., $309.00 €$ Є. ISBN 978-0-470-01671-8

Der neue Band aus der Reihe „Metal Ions in Life Sciences" ist ein umfassendes Nachschlagewerk, in dem das aktuelle Wissen über die Biogeochemie, Biologie, Chemie und Biochemie des Nickels zusammengefasst ist. Die 17 Kapitel des Buchs, die von insgesamt 47 Autoren geschrieben wurden, sind zumeist auf übersichtliche und prägnante Weise dargestellt, und nur in wenigen Ausnahmen gibt es Überschneidungen mit anderen Kapiteln. Das Buch ist vorwiegend für Wissenschaftler konzipiert, die die Rolle von Nickel in der Biologie erforschen.

In Kapitel 1 werden die Biogeochemie und die interessante Geschichte des Nickels behandelt, wobei auch auf Anwendungen und die Bioverfügbarkeit eingegangen wird. Kapitel 2 beschäftigt sich mit den biologischen Eigenschaften und der Toxizität von Nickel. Die Resorption, die Metabolisierung und die Ausscheidung von Nickel durch Organismen werden erläutert. Außerdem werden Methoden der Bestimmung von Nickelkonzentrationen und damit verbundene Probleme erörtert. Eine interessante Diskussion über die Beweglichkeit und Bioverfügbarkeit von Nickel schließt sich an. Die beiden ersten Kapitel mit ihren nahezu 400 Literaturhinweisen liefern eine profunde Grundlage für das Verständnis der nachfolgenden, spezielleren Themen.

Die Kapitel 3 und 4 sind der bioanorganischen Chemie des Nickels gewidmet. Unter anderem werden $\mathrm{Ni}$ ckelkomplexe mit Aminosäuren, Peptiden, Kohlenhydraten und Nucleotiden beschrieben. Fast 500 Hinweise auf einschlägige Publikationen sind hier $\mathrm{zu}$ finden. Beeindruckend ist die Beschreibung von komplexen Vielkomponentensystemen in einer kontrollierbaren Lösung, wenn auch die Komplexität in der lebenden Zelle, in der unterschiedliche Metallionen in die entsprechenden aktiven Zentren eines Proteins integriert werden, noch weitaus höher ist. Die Ausführungen tragen viel zum Verständnis der Metallkomplexierung in der Biologie bei.

Kapitel 5 ist eine detaillierte Abhandlung über Modelle aktiver Zentren in nickelhaltigen Proteinen. Die Ausführungen zur biomimetischen Chemie sind eine vortreffliche Ergänzung des ausgezeichneten Übersichtsartikels von Halcrow und Christou über Nickel-Modellkomplexe aus dem Jahr 1994 (Chem. Rev. 1994, 94, 2421-2476). Die Aktualisierungen beziehen sich auf wichtige Forschungen nach der Kristallstrukturaufklärung nickelhaltiger Enzyme, die teilweise neue, unerwartete Koordinationssphären aufwiesen. Diese Beschreibung der aktiven Zentren unter dem Aspekt der Koordinationschemie und der Herausforderungen bei der Synthese von Struktur- und Funktionsmodellen dieser Zentren ist eindrucksvoll gelungen.

Es folgen sieben Kapitel zum allgemeinen Thema nickelbasierte Enzymologie. Eine Diskussion über Strukturen von Ureasen in Kapitel 6 eröffnet diesen Themenbereich. Verschiedene Addukte von Ureasen mit Metallen, Substraten und Inhibitoren, die kristallin erhalten werden konnten, werden vorgestellt. Für Biochemiker, die sich intensiv mit Nickel beschäftigen, gehört Kapitel 7 über Ni-Fe-Hydrogenasen zur Pflichtlektüre. Unter anderem werden hier Ergebnisse gentechnischer, röntgenographischer und spektroskopischer Untersuchungen vieler Forschungsgruppen aus den letzten zehn Jahren präsentiert.
Außerdem bietet dieser exzellente Beitrag eine Übersicht über kontrovers diskutierte Themen und ungeklärte Probleme. In Kapitel 8 stellen die Autoren ihre wichtigsten Arbeiten der letzten zehn Jahre über die Methylcoenzym-M-Reduktase und das Coenzym $\mathrm{F}_{430}$ vor. In Kapitel 9 werden die unterschiedlichen Theorien erörtert, die hinsichtlich der Acetyl-CoA-Synthase (ACS) und den nickelhaltigen CO-Dehydrogenasen (CODHs) bestehen. Wenn die Autoren, wie hier geschehen, versuchen, die verschiedenen Theorien miteinander in Einklang zu bringen, hätte man meines Erachtens die unterschiedlichen Ansichten ausgewogener darstellen sollen. Umfassende, prägnante Informationen findet der Leser im Kapitel über Superoxiddismutase. Es werden interessante Themen wie der „nickel hook“ - eine ungewöhnliche Nickelkoordination - und seine Rolle für die Einstellung des Redoxpotentials, die Bedeutung des proteolytischen Verfahrens für die Erzeugung des aktiven Proteins sowie mögliche Mechanismen des Protonentransfers beschrieben. In den beiden folgenden Kapiteln stehen die Enzyme Glyoxylase I bzw. Aciredukton-Dioxygenase, in denen Nickel durch andere Metalle ersetzt werden kann, im Mittelpunkt. In Kapitel 11 wird eine Glyoxylase I, in der neben Nickel andere Metallionen inkorporiert sind, als die vielleicht aktivste Form dieses Enzyms präsentiert. Während die Glyoxylase I, ganz gleich welche Metallionen inkorporiert sind, die Bildung der gleichen Produkte katalysiert, hängt, wie in Kapitel 12 gezeigt wird, bei der Aciredukton-Dioxygenase, einem Mitglied der Cupin-Familie, die Produktverteilung davon ab, ob Nickel oder Eisen im aktiven Zentrum koordiniert ist. Dieses Enzym ist von besonderem biomedizinischem Interesse, denn bisher ist noch nicht bekannt, ob das menschliche Enzym Nickel, Eisen oder vielleicht ein anderes Metallion enthält. Es könnte das erste nickelhaltige Enzym sein, das im menschlichen Organismus entdeckt wird. Als Quelle von CO, das als wichtiges Signalmolekül erkannt wurde, könnte es die Funktion der HämOxygenase ergänzen.

Die letzten fünf Kapitel liefern wichtige Informationen über die Inkorporation von Nickel in Metallzentren 
von Proteinen, die nickelabhängige Genexpression, die Rolle von Nickel bei Erkrankungen und über toxikologische Eigenschaften des Nickels. In Kapitel 13 wird über SlyD berichtet, wobei Arbeiten über dieses Protein bis 2005 berücksichtigt werden. Die Verbindung zum Nickelmetabolismus wird im Beitrag allerdings erst spät ersichtlich. AuBerdem konnten wichtige aktuelle Forschungsergebnisse von Zamble wohl aus zeitlichen Gründen nicht mehr aufgenommen werden. Nach Zamble ist SlyD ein molekulares Chaperon für Hydrogenase. Zudem hat er festgestellt, dass die cis-trans-Prolyl-Isomeraseaktivität von SlyD bei der Reifung der Hydrogenase keine Rolle spielt. Neben einem achtseitigen flüchtigen Exkurs über Nickelenzyme, die in den Kapiteln 7-12 bereits ausführlich abgehandelt werden, bietet Kapitel 14 wichtige und aktuelle Informationen über Metallchaperone wie UreE, HypAb, SlyD und CooJ, die Nickel anlagern, und molekulare Chaperone wie UreDFG, HypBC, CooC und AcsF, die die Bildung von Nickelzentren in Urease, Hydrogenase und CO-Dehydrogenase unterstützen. Während in Kapitel 6 Strukturen von Ureasen detailliert erörtert werden, stehen in Kapitel 15 der Erreger Helicobacter pylori und die Bedeutung von Urease und Nickel für die Biologie und Pathogenität dieses Organismus im Mittelpunkt. Wichtige Themen wie Metallion-Homöostase, Regulierung und katalytische Aktivität in Organismen werden ausgezeichnet beschrieben. In Kapitel 15 wird auch die nickelabhängige Genexpression durch NikR behandelt. Dieses interessante Thema hätte man jedoch eher in Kapitel 16, das speziell der nickelabhängigen Genexpression gewidmet ist, erwartet. Hier wird indes die durch Nickelanlagerung veränderte Genexpression in Säugerzellen unter dem Aspekt krankhafter Veränderungen erörtert. Die mit erhöhten und verminderten Konzentrationen in der Umwelt verbundene Toxikologie und Pathogenität von Nickel wird in Kapitel 17 abgehandelt. Neben anderen interessanten Themen werden die Beziehung zwischen der Redoxaktivität von Nickelkomplexen mit verschiedenen zellständigen Liganden und der Toxizität sowie der offensichtliche Effekt der Nickelexposition auf die Selektion der Zellen mit einer hohen Glycolysegeschwindigkeit (z.B. Krebszellen) behandelt.

Das Buch kann allen Wissenschaftlern, die sich für die Rolle von Metallen und insbesondere des Nickels in der Biologie interessieren, empfohlen werden.

\section{Stephen W. Ragsdale}

Department of Biological Chemistry University of Michigan Medical School Ann Arbor (USA)

\section{DOI: $10.1002 /$ ange.200785510}

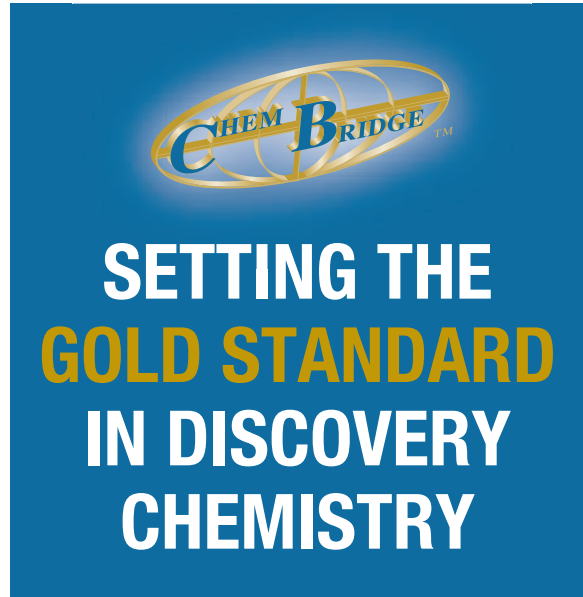

\section{PORTFOLIO \\ - 700,000 \\ Screening \\ Compounds \\ - Hit2Lead.com \\ e-Commerce \\ Site \\ SEARCH, \\ FIND, \\ BUY}

EXPERIENCE

- 15 Years of

excellence in

chemistry

products \&

research

services

$\bullet 185$

Chemists:

synthetic,

high-

throughput,

\& medicinal

SUCGESS

- Major, multi-year

alliances with top

pharmaceutical

$\&$ biotech

companies

- High Quality

Control

Standards \&

Compound

Purity

- 400 Clients

World Wide:

pharma, biotech,

academic, \&

non-profit

CHEMBRIDGE CORPORATION IS THE LEADING

GLOBAL DISCOVERY CHEMISTRY CRO AND

PREMIER PROVIDER OF ADVANCED SCREEN-

ING LIBRARIES FOR SMALL MOLECULE DRUG

DISCOVERY.

1-800-964-6143 I sales@chembridge.com 